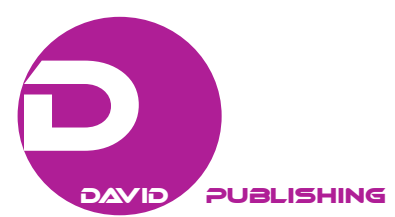

\title{
Some Remarks About Financial Market Modelling Using a Minority Game Approach
}

\author{
Ewa Drabik, Piotr Młodzianowski \\ Warsaw University of Technology, Warsaw, Poland
}

\begin{abstract}
The methods adopted by static physics corroborating the existence of electromagnetic forces are applicable to the theory of financial markets. Perceived from a classically physical angle, the financial market is defined as a system composed of several individual entries cooperating upon electromagnetic principles. The approach concerned gives rise to a certain model of financial market, otherwise known as a minority game. In the case of minority game, the allocation of securities and funds is conditioned exclusively upon the fluctuation of prices, where a higher tendency to purchase goods and stocks results in the scale being more profitable and vice versa. Thus players from a minority group gain a prevailing position.
\end{abstract}

Keywords: modelling of financial markets, minority game, El Farol bar problem

\section{Introduction}

A variety of notions derived from the field of classical physics have found their applications in the realm of economy and social science, for instance the law of conservation energy and the first law of thermodynamics. According to the law of conservation energy, the energy of a body or a system of entities may be neither created nor destroyed without a trace. One type of energy may merely convert into another. The increase of the system's interval energy shall be synopsized as the difference between the energy supplied to the system and the energy released by the system to the environment.

Over the last decade, the above laws have been nearly intuitively applied in economics, predominantly for the purposes of describing economic equilibrium, the concept of which has not been captured within one incontestable definition. In the overarching variety of cases, it is used to denote the balance between supply and demand for all the goods (general equilibrium) or specific goods (partial equilibrium). Analyzed from a more general perspective, equilibrium, and the forces affecting economic systems can be expressed in a number of ways within the area of economic theory. Mostly, a position of equilibrium is a minimal potential and kinetic energy.

A number of economic models adhering to the basic laws of physics have been known since 1960s, with a particular emphasis placed upon the law of gravity. Material points were replaced by "centers" such as cities, shopping malls, and the term "gravitation" was replaced by its economic equivalent i.e., interaction. Physical mass is credited as the total of such variables as households, consumption cities, hospital beds, newspapers,

Ewa Drabik, professor, Faculty of Management, Warsaw University of Technology, Warsaw, Poland.

Piotr Młodzianowski, master of science, Faculty of Management, Warsaw University of Technology, Warsaw, Poland.

Correspondence concerning this article should be addressed to Ewa Drabik, Faculty of Management, Warsaw University of Technology, Narbutta 85, 02-524 Warsaw, Poland. 
magazine circulation, population, etc. Distance is measured in kilometers, time of travel, or quotient of the product price. Gravitation models describing spatial economic interaction were proposed by W. Reilly, R. Bachi, T. Stanley, P. D. Convers, D. L. Huff, and P. Krugman (Drabik, 2011).

The model of minority and El Farol Bar problem are presented in chapter 2. The numerical results of study of some minority game on study of some minority game on financial markets are presented in chapter 3. Finally, in chapter 4 , some informations about quantum games are referred.

\section{Research Design}

\section{Research Subject}

The paper aims at demonstrating the way in which the data gathered from the specialist internet websites dedicated to the stock exchange affect the stock index forecast prepared by means of the IMG model.

\section{Definition of Minority Games and Its Economic Application}

The last decade saw the rise in popularity of a financial market model called the minority game (MG), which operates in a fashion similar to a macroscopic system in static physics. Market participants whose number proves to be high (they are often referred to as a "continuum") buy or sell securities. They rely on the analysis of the stock price fluctuation and other information they receive. If the majority of market participants intend to buy a stock, the sale proves reasonable and justifiable, and vice versa. Minority group participants usually win. All market participants make decisions based on their experience and certain information pattern.

The research on the minority game was initiated by Arthur (1992) where the El Farol bar problem was defined. The motivation came from studying congestion in the bar in Santa Fe, New Mexico. Every week people contemplated over whether it was worth showing there up or not. As the space in the bar was limited, it was favorable if only a few show up. It was necessary for everyone to decide concurrently whether they would go to the bar or not, assuming the individuals do not communicate. The El Farol problem lies in the definition of players' strategies. A strategy in this problem is a rule which "predicts" the attendance of the next week, given the information about the attendance in the previous week.

There are three types of participants: (1) players who create information noise and make random decisions; (2) producers who behave in a deterministic way; and (3) speculators who seek profit using all methods available. The activities of all these groups cumulate and cause a qualitative change in the entire system, as in the case of typical macroscopic systems where electromagnetic impacts are observed on a macro scale. From the point of view of a market participant, it is essential to identify such parameters of the system's status which characterize the system status on a macro scale. Because there are large numbers of participants, the law of large numbers is often used (Challet \& Zhang 1998).

Minority game may be described as follows:

(1) There are sets of I market participants-players.

(2) At each time step $t(t=1,2, \ldots)$ the $i$-th player out of $i(i=1,2, \ldots N)$ takes action.

$a_{i}(t)=+1$ or $a_{i}(t)=-1$ according to some strategy: $s_{i}(t):\{1, \ldots, \mu, \ldots, P\} \rightarrow\{-1 ;+1\}$, where $\{1, \ldots, \mu, \ldots, P\}$ is an information set. The players who play $a_{i}(t)=-1$ will win and those who choose $a_{i}(t)=+1$ will lose ("buy" or "sell"). Information "includes" both the game history, or a string of minority and majority players decision in the past and certain information received from outside.

(3) The gain and all "aggregated demand" is defined. 


$$
g_{i}(t)=-a_{i}(t) A(t) \text { where } A(t)=\sum_{j=1}^{N} a_{j}(t)
$$

where $A(t)$ denotes the difference between the number of players who decided to sell and the number of players who buy a stock at time $t$.

(4) The minority action $a *(t)$ is determined from $A(t)$ i.e. $a *(t)=-\operatorname{sgn} A(t)$.

(5) The aim of each market participant is profit maximization $g_{i}(t)(i=1, \ldots, N)$ at any stage of game $t$. The problem can be solved in a number of ways (Challet \& Zhang, 1998; Arthur, Holland, Lebron, \& Palmer, 1994).

Financial market can be compared along with its prices, transactions, assets, money, etc., with the minority game. But the minority game describes markets only under a certain assumption. In minority game, fluctuation phenomena and their statistical properties can be studied. Statistical physics suggests that the collective behavior of a system at many interacting units is a highly simplified description on the microscale. A major emphasis is placed in a market for a single asset and call $p(t)$ its price at time $t$. The price is assumed to be the difference between the number of shares being bought and sold. Financial markets concentrated on a minority game modelling have also focused examination function $A(t)$. Several price formation rules, which link the excess demand $A(t)$ to price return $r(t)$, have been proposed in literature (Farmer, 1999) and used in the minority game context. The price return $r(t)$ depends linearly on $A(t)$

$$
r(t) \equiv \log p(t)-\log p(t-1)=\frac{A(t)}{\lambda}
$$

where $\lambda$ is called the liquidity or market depth and

$$
\log (p(t)) \equiv \sum_{t^{\prime} \leq t}^{t} \frac{A\left(t^{\prime}\right)}{\lambda}
$$

The variable that is also examined is $a=\frac{P}{N}$. When $a>>1$, there is too much information circulating in the market and players act randomly. When $a \rightarrow 1$ more players join the game and the game and the information received is used more efficiently. When $\alpha$ researches its minimum $\left(a=\frac{7}{20}\right)$ the behavior market participants can be compared to the description of critical phenomena in static physics.

\section{Review of Problems and Literatures Relationship With MG}

Another interesting ratio in MG-problem is a control parameter. Savit, Manucca, and Riolo (1999) found that fluctuation of attendance depends on the ratio between the number of players $N$ and the number of possible histories in the game, $2^{\mathrm{m}}: N / 2^{\mathrm{m}}$. This ratio is called that the game is in a crowded phase or in a heard regime. When the strategy space is much larger than the number of players, the game is random regime. In this regime, the strategies are similar, thus players behave mostly randomly and the game may be constructed as Random Choice Game (RCG). In MG literature, RCG is the game with mixed strategies. Geometrical analysis of the strategy space inspired Challet and Zhang (1998) to introduce the Reduced Strategy Space (RSS). The RSS includes only $2^{\mathrm{m}+1}$ strategies. 
Another useful concept, the predictability, was also introduced by Savit, Manucca and Riolo (1999). In this case, there are two phases of MG: the asymmetric and symmetric one. The game is in an asymmetric phase if the conditional distribution of minority action is asymmetric around zero and if actions are assumed to take the values: +1 or -1 . The game is in this phase if the players are less relative to $2^{\mathrm{m}}$ possible histories. In a symmetric phase, some information is carried by past outcomes i.e., assuming that a longer data history is analyzed. Johnson, Hart, and Hui (1999) introduced the Crowd-Anticrowd theory. Players act as a crowd if they use the same strategy. If there is a group that simultaneously uses the anti-correlated strategy, they make the opposite decision and hence are considered as an anticrowd. If the sizes of crowd and anticrowd are similar, the cooperation regime takes place. If the crowd dominates majority of players to behave in the same manner, MG can be also interpreted as a stochastically distributed deterministic system. The behavior of the game is dictated by realization of distribution of players over strategies and not just by specific game parameters.

There is a number of papers where authors postulate a coexistence of players with various payoffs. Marsili (2001) pointed out that players' behavior in the minority game reflects only fundamentalist (contrarians) approach while on the markets there are two types of investors: the contrarians and trend-followers. The term "contrarians" stands for the group of investors fading a move positioning against a move or trend in the market.

The authors assumed that the markets are either minority or majority games depending on the ratio between fundamentalists and trend-followers acting in the market. The payoff function changes the investor's profile in that way that he prefers to be in majority. If the number of trend-followers exceeds the number of contrarians, an explosion of global fluctuations appears.

In the former approach, investors do not act if they find their strategies potentiallly unprofitable. This group of models is known as grand canonical MG (Slanina \& Zhang, 1999; Johnson, Hart, Hui, \& Zhang, 2000). The name is borrowed from the statistical mechanics where statistical ensembles with a variable number of particles are called: Grand Canonical. Such kind of game exhibits relatively large fluctuations of aggregate demand. The large price movements exist because players with similar unprofitable strategies jump into the market at the same time (Wawrzyniak, 2011). There are some other MG-like models, mostly much more complicated than the original one, that address financial market, e.g., the Santa Fe artifical market was a trailblazer of multi-players financial models with a variety of trading strategies. In this case, the players are assumed to possess different strategies. The price is a function to the aggregate demand and individuals' wealth varies in time. The game exhibits three regimes: intermitted, oscillating, and stable.

\section{Research Findings}

\section{Some Financial Model of MG}

The behaviour of stock indexes is determined by participants of the market: buyers and sellers. Shaped by a ratio between a number of stocks offered to sell and declared to be purchased, the final price of a stock may be construed as the classic game of demand and supply, defining a point of balance during a transaction. Investment decisions depend upon a variety of factor i.e., strategy, personality of an investor, risk aversion, or information environment which is often the only source of information about a company or state of the national and world economy, especially for small investors.

The study ventured to answer the following questions: 
Does the information environment represented by the articles published on the most popular Polish websites devoted to the field of finance, reflect the behaviour of Warsaw Stock Index (WSI)?

Does the information environment affect the behaviour of Warsaw Stock Index on the next day of exchange quotation?

For the purposes of the study, information environment was classified as positive or negative, according to frequency of positive and negative key words occurring in the article's content. The classification was conducted in compliance with the ensuing formula:

$$
I_{e}=W_{b}-W_{f}
$$

where:

$I_{e}$-information environment;

$W_{b}$-number of key words describing boom in stock market;

$W_{f}$ - number of key words describing fall in stock market.

The result of the formula can be interpreted as:

$I_{e}>0$-positive environment-boom;

$I_{e}<0$-negative environment-fall.

Information environment analysis was performed daily over the period of 55 days of exchange quotation, at the same time after the session. The obtained information was contingent upon the stock index behavior, whereas the forecast was estimated for the next day of exchange quotation (except for Monday for which the information environment analysis was performed on Sunday). When WSI decreases at the closing of the session concerned, information environment should be considered negative; upon the WSI increase it should be specified as positive. The results of the research is shown in Figure 1.

The result of information environment analysis explains the WSI change in $72 \%$, which was expressed with points (green line). The WSI change direction was depicted with $60 \%$ accuracy. It means that in $60 \%$ of cases, the WSI decrease and increase were consistent with the results of information environment analysis carried out after the session ending. The value of an unexplained WSI change equaled $28 \%$ and was observed after sessions featured by relatively low variability. During 55 sessions, the WSI value lost 1,461 points, increasing in 30 sessions and decreasing in 25 sessions. An in-depth analysis of the most important data revealed the correlation between the WSI behavior and information environment. However, the time of observation proved too short to summarize it as a stable phenomenon.

The influence of information environment on the future value of WSI was examined based on an opening and closing exchange of the index, so in a different way comparing to the first study question (reference exchange applicable to the previous day and closing exchange applicable to the current day). The difference was caused by the fact that the information environment analysis was conducted after a session, therefore, it was not possible to take a position in WSI until an exchange quotation opening on the consecutive day. Figure 2 shows the results of the analysis. 


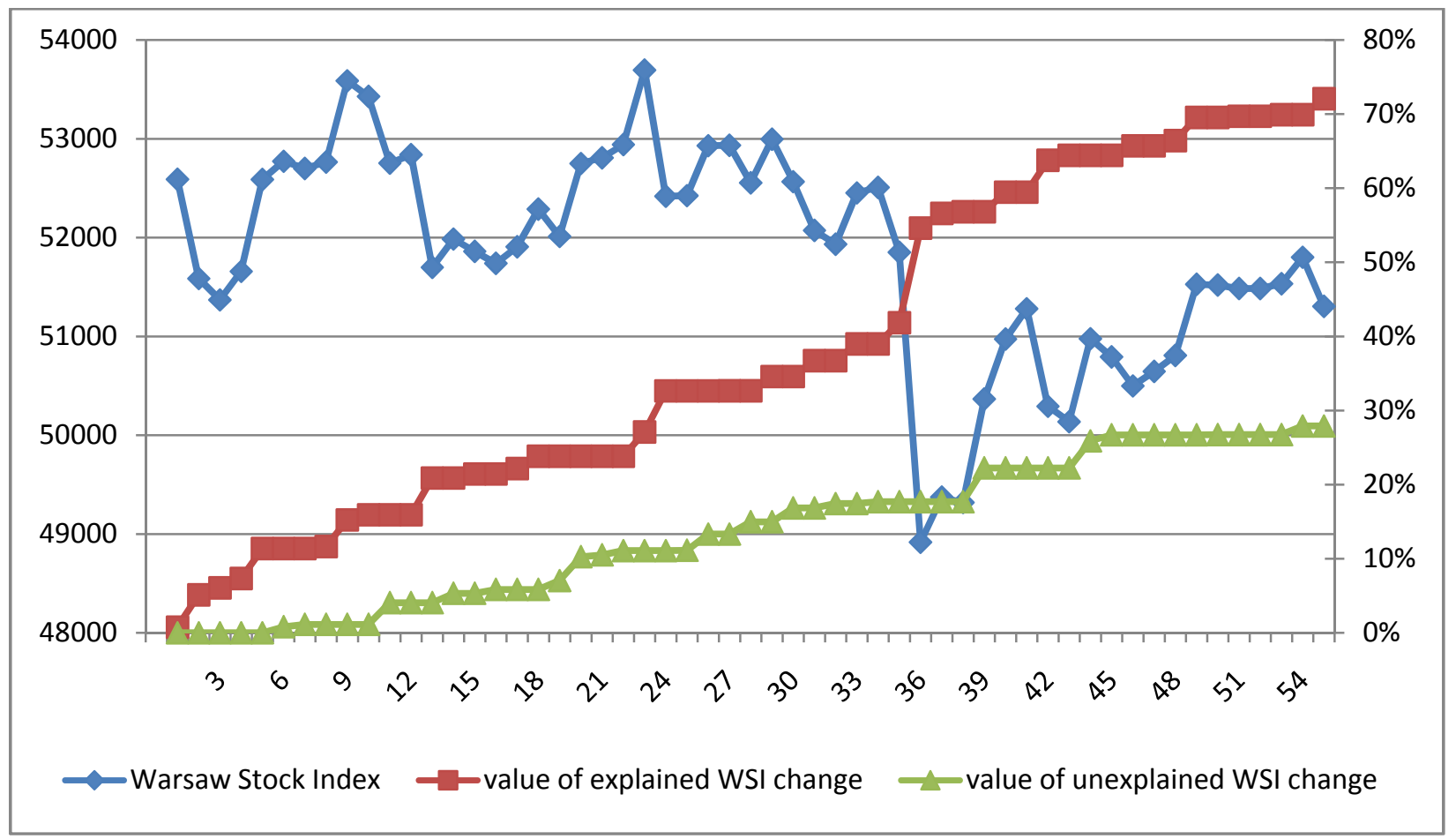

Figure 1. The influence of information environment on WSI change. Source: Economics news about Warsaw Stock Exchange (2015a; 2015b; 2015c; 2015d).

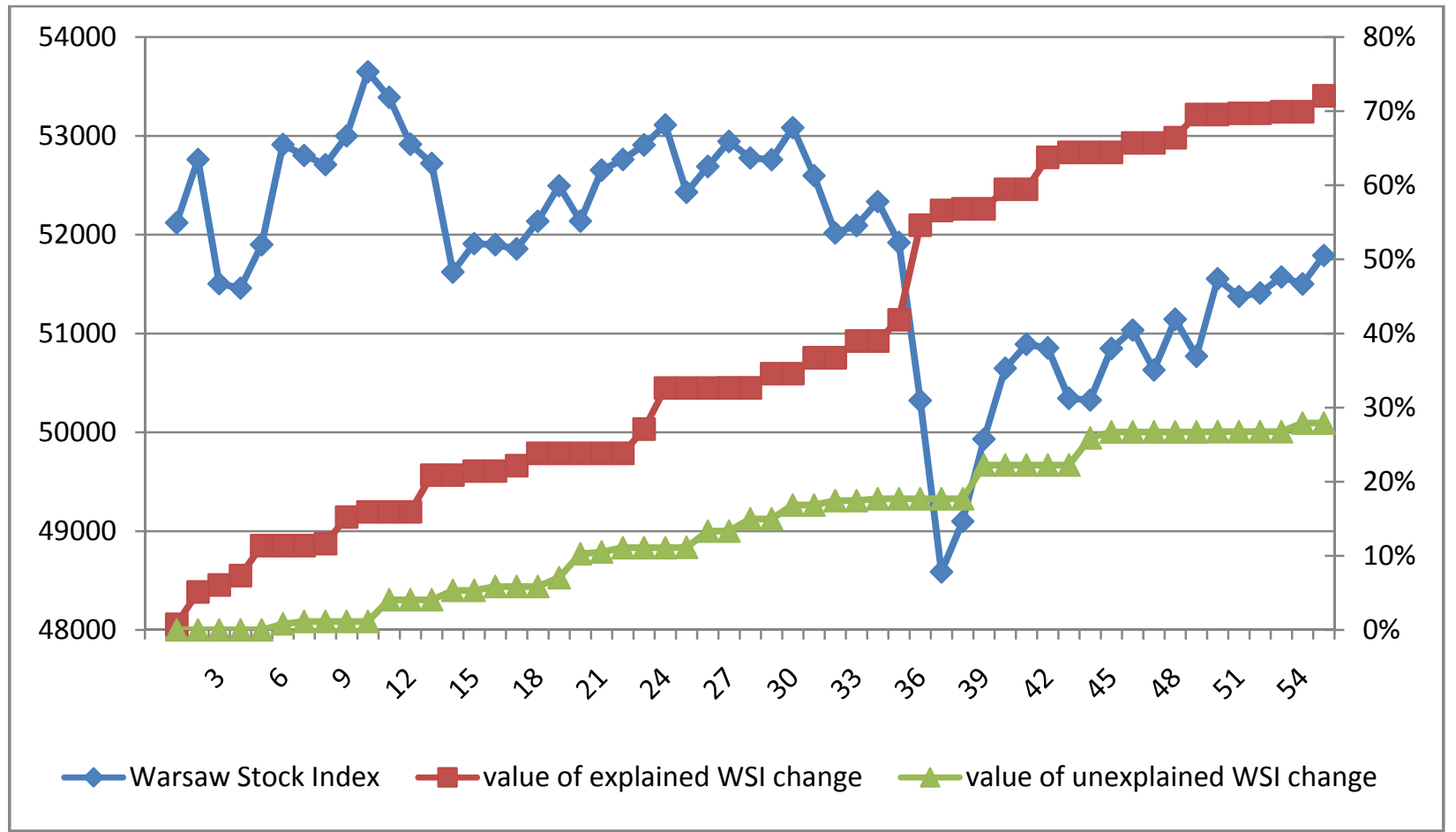

Figure 2. The prediction of WSI change based on the information environment analysis. Source: Economics news about Warsaw Stock Exchange (2015a; 2015b; 2015c; 2015d; 2015e; 2015f; 2015g). 
The nature of an information environment in $61 \%$ of cases was consistent with the WSI change direction on the next day of exchange quotation. It explains $56 \%$ of the WSI change. During 55 sessions, the complete prediction result had a negative value only once and the analysis ended with a result of 2,179 points. It is hard to assess whether the information environment may predict the WSI behaviour or not. The performance of an analysis immediately before opening an exchange quotation may improve the results obtained. The prospective research may take into account information from the USA and Asia, which often affects the stock market in Poland.

\section{Another Applications of Physics Models in Economy}

The quantum game is another economic model that applies the laws of physics.

Game theory is the study of a decision making process in conflict situations. It seems to have asked for a quantum version. Quantum game theory began with works of Wiesner (1983) on quantum money. Eisert, Wilkens, and Lewstein (1999) pioneered in formulating their "quantum problems" in the game formalization. Quantum mechanics is a theory describing the behavior of elementary particles on an atomic scale, where the macro scale still remains applicable.

In particular, a two $\mathfrak{I}$ of the physical system, the initial state $\rho \in S(\mathfrak{I})$, where $S(\mathfrak{I})$ is associated state space, the sets $S_{A}$ and $S_{B}$ of permissible quantum operations of the two players and the pay-off (utility) function $U_{A}$ and $U_{B}$ specify the pay-off of each player. A quantum strategy $s_{A} \in S_{A}$ and $s_{B} \in S_{B}$ is a collection of admissible quantum operations, that is mapping of the space of states onto itself. The quantum game's definition may also include certain additional rules, such as the order of the implementation of the respective quantum strategies or restriction on the admissible communication channels, methods of stopping the game, etc. Quantum game theory has found various applications in physics, for instance quantum strategies will being qubits. The quantum game theory may be suitable for the description of market transactions. There are some analogies with quantum thermodynamics that allow interpreting market equilibrium as the state with a financial risk flow. Euphoria, panic, or herd instinct often cause violent changes of market prices. In general, the quantum description of market phenomena may be more accurate than classical.

\section{Conclusions}

The modelling of economic phenomena based on classical physics and quantum theory provides a more precise description than the classical probability calculus. The game theory featured by the study of a decision making process in conflict circumstances has its quantum theory-related counterpart. In the overarching majority of the analyzed cases, the quantum description of the system outweighs the advantages of classical characteristics. Many physical aspects of information characterize to some economical research in quantum communication.

The empirical part attempts to prove that the minority game model, where the information inflow is taken into consideration to a satisfying degree, impacts the stock index forecast. The research will be continued in the future.

\section{References}

Arthur, W. B. (1992). On learning and adaptation in the economy. Santa Fe Institute Paper, 92, 7-38.

Arthur, W. B., Hollad, J. H., Lebaron, B., \& Palmer, R. (1994). Artificial economic life: A simple model of stock market. Physica D, 25, 264-274. 
Challet, D., \& Zhang, Y.-C. (1998). On the minority games. Analytical and numerical studies. Physica A, 256A, 514-532.

Drabik, E. (2011). On certain analogies between the laws of quantum mechanics and rules of an English auction. Folia Oeconomica Stetinensia, 10(18), 219-237.

Eisert, J., Wilkens, M., \& Lewstein, M. (1999). Quantum games and quantum strategies. Physical Review Letters, 83, 3077.

Economics news about Warsaw Stock Exchange. (2015a July-October). Retrieved from http://biznes.onet.pl

Economics news about Warsaw Stock Exchange. (2015b July-October). Retrieved from http://finanse.wnp.pl

Economics news about Warsaw Stock Exchange. (2015c July-October). Retrieved from http://wyborcza.biz/Gieldy/0,0.html

Economics news about Warsaw Stock Exchange. (2015d July-October). Retrieved from http://www.money.pl

Economics news about Warsaw Stock Exchange. (2015e July-October). Retrieved from http://biznes.interia.pl/wiadomosci-dnia

Economics news about Warsaw Stock Exchange. (2015f July-October). Retrieved from http://www.bankier.pl

Economics news about Warsaw Stock Exchange. (2015g July-October). Retrieved from http://finanse.wp.pl

Farmer, J. D. (1999). Physicists attempt to scale the ivory towers of finance. Computing in Science and Engineering, 1, $26-39$.

Johnson, N. F., Hart, M., \& Hui, P. M. (1999). Crowd effect and volatility in markets with competing agents. Physica, 269 , 1-8.

Johnson, N. F., Hart, M., Hui, P. M., \& Zhang, Y. D. (2000). Trader dynamics in a model market. Journal of Theoretical and Applied Finance, 3, 443-459.

Marsili, M. (2001). Market mechanism and expectations in minority and majority fames. Physica A, 299, 93-103.

Savit, R., Manucca, R., \& Riolo, R. (1999). Adaptive competition market efficiency and phase transition. Physical Review Letters, 82, 2203-2206.

Slanina, F., \& Zhang, Y. G. (1999). Capital flow in two-Component dynamical system. Physica A, 272, 157-268.

Wawrzyniak, K. (2011). On phenomenology, dynamics and some applications of the minority game. (Ph.D. dissertation, Polish Academy of Science) (in Poland).

Wiesner, S. (1983). Conjugate coding. SIGACT News, 15(1), 78. 